\title{
Colorectal Adenocarcinoma Post-Operative Treatment with Chinese Herbs
}

\author{
Jihe Zhu1, Blagica Arsovska ${ }^{1,2}$, Kristina Kozovska1 ${ }^{*}$ \\ ${ }^{1}$ Faculty of Medical Sciences, Goce Delčev University of Štip, Štip, Republic of Macedonia \\ ${ }^{2}$ Institute of Biology, Faculty of Natural Sciences and Mathematics, Skopje, Republic of Macedonia \\ Email: *tongdatang-tcm@hotmail.com
}

How to cite this paper: Zhu, J., Arsovska, B. and Kozovska, K. (2018) Colorectal Adenocarcinoma Post-Operative Treatment with Chinese Herbs. Yangtze Medicine, 2, 46-50.

https://doi.org/10.4236/ym.2018.21005

Received: November 8, 2017

Accepted: March 24, 2018

Published: March 27, 2018

Copyright (c) 2018 by authors and Scientific Research Publishing Inc. This work is licensed under the Creative Commons Attribution International License (CC BY 4.0).

http://creativecommons.org/licenses/by/4.0/

\section{cc) (i) Open Access}

\begin{abstract}
Colorectal cancer is malignancy of the colon or rectum. Adenocarcinoma is the most common colorectal cancer. These malignant cancer cells grow inside the colon and the rectum. Large clusters of these cells form structures known as tumors. Our treated patient is a 48 year old male who has undergone colorectal adenocarcinoma surgery in stage IIIB. During the surgery he was resected part of the colon with a total length of $57 \mathrm{~cm}$. We started the treatment with Chinese herbs immediately after the surgery with no additional chemotherapy and radiotherapy. Herbs used in the treatment are Cordyceps sinensis, Ganoderma broken spores (Reishi) and Yunnan Baiyao. Before the surgery the patient was pale, exhausted, with abdominal pain on the left side under the ribs, Iron ( $\mathrm{Fe}$ ) was very low-7, tumor marker (CEA) was high-39.64 ng/mL, hematochezia was present and with insulin intake of 34 units (the patient is diabetic). Two months after using the herbs daily, the patient blood analysis was improved, Iron ( $\mathrm{Fe}$ ) is elevated, no hematochezia is detected, organs are with normal findings, normal color of the face is restored, post-operative scar healed very well and quickly and insulin intake is reduced from 34 to 6 units. After 6 months of treatment the results are amazing, the patient is in excellent health condition, the treatment is completed and is no longer taking any medications. With the herbal treatment we succeeded to keep the patient in good health, boost the immune system and improve the Qi energy flow.
\end{abstract}

\section{Keywords}

Treatment, Traditional Chinese Medicine, Cancer, Adenocarcinoma, Colon

\section{Introduction}

Colorectal cancer is malignancy of the colon or rectum. Colon cancer is the third most commonly diagnosed cancer in men and women in US and the second 
leading cause of cancer-related deaths. Colorectal cancer occurs in the last 1.8 meters of the intestine (colon and rectum). Adenocarcinoma is the most common colorectal cancer. These malignant cancer cells grow inside the colon and the rectum. They originate from the epithelial cells of the colorectal mucosa. Large clusters of these cells form structures known as tumors [1] [2]. Main causes and risk factors for colorectal adenocarcinoma are: genetic factors, dietary factors (diet high in animal fat and red meat), obesity, passive lifestyle, inflammatory bowel diseases, polyps of colon, night work, etc. Symptoms that may appear are: abdominal pain, rectal bleeding, diarrhea, constipation, iron deficiency anemia, decreased appetite, weight loss, extreme fatigue, etc. [1] [3]. Early diagnosis is critical for successful treatment of colorectal cancer. Procedures that may be done to confirm the diagnosis are endoscopic biopsy, sigmoidoscopy, colonoscopy, abdominal CT, complete blood count, etc. The treatment options are surgery, chemotherapy, herbal treatment and as a prevention proper nutrition and active lifestyle [1] [2].

Herbal treatment and herbal formulas as part of the Traditional Chinese Medicine (TCM) are very often used as a treatment in variety of malignancies. The herbs that are used in the treatment have anticancer activities and can induce the apoptosis of cancer cells. Herbal treatment can help to improve the survival of cancer patients, enhance the quality of life, reduce the side-effects and toxic effects from the radiotherapy and chemotherapy, promote the function of the immune system, suppress the inflammation, reduce the cancer occurrence and recurrence, regulate the motility and adhesion of the cancer cells, show anti-angiogenic effects, etc. [4] [5].

From a TCM perspective, colorectal cancer occurs due to toxicity accumulation, Qi and blood deficiency, Yin/Yang deficiency, Liver, Kidney, Spleen and/or Stomach Qi deficiency, Phlegm-Dampness and/or Damp-Heat presence. If Yin deficiency is present the survival time is shorter. If Qi deficiency is present, the survival time is longer. Most common syndrome is Spleen and Kidney Yang deficiency [5]. Therefore, the treatment aim is to balance the energy in the affected area, balance the Yin and Yang, promote healthy fresh blood and Qi, eliminate the toxins, expel all the pathogenic factors like Damp and Heat, improve the immune system, etc.

\section{Methods and Results}

The treated patient is a 48 year old male who have undergone colorectal adenocarcinoma surgery in stage IIIB. The patient is diabetic, with normal appetite, variable blood pressure (Verapamil was taken occasionally), insomnia and increased sweating. Before the surgery the patient was pale, exhausted, with abdominal pain on the left side under the ribs, Iron ( $\mathrm{Fe}$ ) was very low, tumor marker (CEA) was high, hematochezia was present and with insulin intake of 34 units. The blood analysis showed high C-reactive protein (CRP) - $19 \mathrm{mg} / \mathrm{l}$ (normal range $0-10$ ), high carcinoembryonic antigen (CEA) $-39.64 \mathrm{ng} / \mathrm{mL}$ (normal 
range < 5.0), low $\mathrm{Hb}-102 \mathrm{~g} / \mathrm{l}$ (normal range 115 - 180), low $\mathrm{Fe}-7$ (normal range 9.0 - 31.0), high K-5.5 (normal range 3.5 - 5.1), high leukocytes $-13.9 \times 10^{-3} \mathrm{ml}$ (normal range $4.0-11.0$ ), high thrombocytes $-698 \times 10^{-3} \mathrm{ml}$ (normal range 150 - 400), low lymphocytes-0.19 (0.21 - 0.25) and urine $\mathrm{pH}-5.0$ (6.5 - 7.5). During the surgery was resected part of the colon with total length of $57 \mathrm{~cm}-$ Caecum with appendix, colon ascendens, colon transfersum and part of colon descendens. The widest part of colic fat tissue was $6 \mathrm{~mm}$. In longitudinal dissection, at $12 \mathrm{~cm}$ from the distal dissection boundary, in the part of the linear flexure, was present an exophytic tumor formation $-9 \mathrm{~cm}$. Tumor formation occupied the entire circumference of the wall of the intestine and obstructed the lumen. Overall, the finding shows moderately differentiated colon adenocarcinoma. The neoplasm is pTNM = pT3 pN1A pMX G2 L1 V1 stage IIIB.

The treatment with Chinese herbs started immediately after the surgery with no additional chemotherapy and radiotherapy. Herbs used in the treatment are Cordyceps sinensis, Ganoderma broken spores (Reishi) and Yunnan Baiyao. Herbs are taken every day-2 g capsule Cordyceps sinensis, 3 g powder Ganoderma broken spores and $0.75 \mathrm{~g}$ capsule Yunnan Baiyao. Two months after using the herbs daily, the patient blood analysis is improved, Iron $(\mathrm{Fe})$ is elevated (13 $\mathrm{mmol} / \mathrm{L})$, no hematochezia is detected, organs are with normal findings, normal color of the face is restored, post-operative scar healed very well and quickly and insulin intake is reduced from 34 to 6 units. The patient continued on with the herbal treatment preventively, to improve more the health condition and boost the immune system. After six months of treatment the last blood test analysis showed the following results: CEA -2.35 (normal range $<5.0$ ), $\mathrm{Fe}-13.3$ (normal range 9.0 - 31.0), $\mathrm{K}-4.14$ (normal range 3.5 - 5.1), urine $\mathrm{pH}-6.5$ (normal range 6.5 - 7.5), cancer antigen 19-9 (CA 19-9)-11.96 U/ml (normal range 0.0 - 37.01), lymphocytes-2.26 $\times 10^{-3} / \mathrm{uL}$ (normal range $2.10-2.50$ ), thrombocytes-288 $\times 10^{-3} \mathrm{ml}$ (normal range $150-400$ ), leukocytes-7.46 $\times 10^{-3}$ $\mathrm{ml}$ (normal range 4.0 - 11.0), A1c WB-38.8 mM/mol (normal range 29.0 42.0). The patient is in excellent health condition, the treatment is complete and is no longer taking any medications.

\section{Discussion}

Colorectal adenocarcinoma is a very serious and life-threatening condition that needs to be taken very seriously and carefully. Cancer treatment is one of the most difficult tasks in medical service. The treatment should always be aimed at saving the patient's life, to improve the quality of life and to prolong the lifespan. The most common treatment regime is combination of chemotherapy and TCM herbs [6]. However, we treated our patient only with TCM herbs, to avoid the harmful and toxic effects from the chemotherapy and radiotherapy. Although the treatment is slow and takes some time to see the positive results, the condition improves continuously, safely and steadily. The herbal treatment aim is to re-establish the system to promote its own capacity to heal itself [6]. TCM 
usually uses combination of herbal formulas. Herbs that are used in the treatment have similar role to the chemotherapy. "Anticancer" herbs have strong ingredients that raise the immune system, support the vital energy, tonify the Qi energy, vitalize and activate the blood, remove blood stasis, strengthen the body resistance, resolve the phlegm and the abnormal masses [7].

Cordyceps sinensis is a fungus that has been proven to be effective in the cancer treatment. Its active component-the cordycepin shows anti-cancer effects, anti-metastatic action and suppresses the invasiveness of the cancer cells [8] [9] [10]. Cordyceps sinensis in the treatment is used to reinforce the Yin and Yang energy and to keep and improve the energy. Ganoderma broken spores is a fungus that's been proven to inhibit the colorectal cancer and suppress the cell migrations and cell adhesions [11]-[16]. Ganoderma goes to Liver Yin meridian, to clean the toxins and boost the immune system. Yunnan Baiyao quickly heals the injuries and has homeostatic and anti-bacterial properties [17]. It is used to improve and support the blood flow and Qi energy, to promote the blood flow, resolve the stagnation and reduce the inflammation [18] [19] [20] [21] [22]. In some cases acupuncture treatment can be added too to help the patients who experience pain, fatigue, hot flushes, dry mouth, nausea and other symptoms [6].

This case report suggests that further studies with more patients are necessary to compare the Chinese herbs with chemotherapy, no treatment or other therapies.

\section{Conclusion}

With the herbal treatment we succeeded to keep the patient in good health, boost the immune system and improve the Qi energy flow without chemotherapy and radiotherapy.

\section{Conflict of Interest Disclosure}

The authors declare that there are not conflicts of interest.

\section{Patient Consent}

Informed patient consent was obtained for the Traditional Chinese Medicine therapy.

\section{References}

[1] Stojanovska, D. (2011) Colorectal Carcinoma (Colon Cancer). http://www.borka.org.mk

[2] Fleming, M., Ravula, S., Tatishchev, F.S. and Wang, L.H. (2012) Colorectal Carcinoma: Pathologic Aspects. Journal of Gastrointestinal Oncology, 3, 153-173.

[3] Dragovich, T. (2017) Colon Cancer. https://emedicine.medscape.com/

[4] Ye, L., et al. (2015) Traditional Chinese Medicine in the Prevention and Treatment of Cancer and Cancer Metastasis. Oncology Letters, 10, 1240-1250. https://doi.org/10.3892/ol.2015.3459

[5] Deng, S., Hu, B. and An, H.M. (2012) Traditional Chinese Medicinal Syndromes and Treatment in Colorectal Cancer. Journal of Cancer Therapy, 3, 888-897. 
https://doi.org/10.4236/jct.2012.326114

[6] Jiang, D., Meng, F.Y., Li, L. and Qu, F. (2016) The Role of Chinese Medicine in Cancer Care-A Critical Review. World Journal of Traditional Chinese Medicine, 2, 68-73. https://doi.org/10.15806/j.issn.2311-8571.2015.0007

[7] Dharmananda, S. (1997) The Treatment of Gastro-Intestinal Cancers with Chinese Medicine. http://www.itmonline.org/

[8] Nakamura, K., Shinozuka, K. and Yoshikawa, N. (2015) Anticancer and Antimetastatic Effects of Cordycepin, an Active Component of Cordyceps sinensis. Journal of Pharmacological Sciences, 127, 53-56. https://doi.org/10.1016/j.jphs.2014.09.001

[9] Ji, N.F., et al. (2011) Polysaccharide of Cordyceps sinensis Enhances Cisplatin Cytotoxicity in Non-Small Cell Lung Cancer H157 Cell Line. Integrative Cancer Therapies, 10, 359-367. https://doi.org/10.1177/1534735410392573

[10] Sanders, K. (2014) Cordyceps Kill Cancer Cells and Boost Immunity. http://www.naturalhealth365.com

[11] Na, K., et al. (2017) Anticarcinogenic Effects of Water Extract of Sporoderm-Broken Spores of Ganoderma lucidum on Colorectal Cancer in Vitro and in Vivo. International Journal of Oncology, 50, 1541-1554.

[12] Silva, D. (2003) Ganoderma lucidum (Reishi) in Cancer Treatment. Integrative Cancer Therapies, 2, 358-364. https://doi.org/10.1177/1534735403259066

[13] Thyagarajan, A., et al. (2010) Triterpenes from Ganoderma lucidum Induce Autophagy in Colon Cancer through the Inhibition of p38 Mitogen-Activated Kinase (p38 MAPK). Nutrition and Cancer, 62, 630-640. https://doi.org/10.1080/01635580903532390

[14] Jedinak, A., Thyagarajan-Sahu, A., Jiang, J. and Sliva, D. (2011) Ganoderma nontriol, a Lanostanoid Triterpene from Ganoderma lucidum, Suppresses Growth of Colon Cancer Cells through B-Catenin Signaling. International Journal of Oncology, 38, 761-767.

[15] Ye, H. and Jensen, A.B. (2017) Ganoderma lucidum on the Inhibition of Colorectal Cancer. Hematology \& Medical Oncology, 2, 1-4. https://doi.org/10.15761/HMO.1000126

[16] Thyagarajan, A., Nguyen, H., Jedinak, A. and Sliva, D. (2008) Inhibition of Colon Cancer Cells by Ganoderma lucidum Triterpenes. Cancer Research, 68, 630-640.

[17] Lotus, S. (2013) Review: Yunnan Baiyao. http://www.sacredlotus.com

[18] Siekman, D. (2010) Yunnan Baiyao “Secret” Ingredients Found on US Websites. http://www.gokunming.com

[19] Zhu, J., Arsovska, B. and Kozovska, K. (2017) Case Report: Acupuncture and Herbal Treatment with Yunnan Baiyao for Hyperhidrosis. IOSR Journal of Dental and Medical Sciences, 16, 80-81.

[20] Dharmananda, S. (2010) Yunnan Paiyao Uses for Injury and Surgery; Gastro-Intestinal, Respiratory, and Urogenital Disorders. http://www.itmonline.org

[21] Salaga, M., Zatorski, H., Sobczak, M., Chen, C. and Fuchna, J. (2014) Chinese Herbal Medicines in the Treatment of IBD and Colorectal Cancer: A Review. Current Treatment Options in Oncology, 15, 405-420. https://doi.org/10.1007/s11864-014-0288-2

[22] Yang, B., et al. (2014) The Efficacy of Yunnan Baiyao on Haemostasis and Antiulcer: A Systematic Review and Meta-Analysis of Randomized Controlled Trials. International Journal of Clinical and Experimental Medicine, 7, 461-482. 